\section{Home Blood Glucose Monitoring}

\author{
R. B. Tattersall \\ General Hospital, Nottingham, England
}

A recent review in this journal summarized the impressive evidence that poor control is one of the major factors responsible for the micro-vascular complications of diabetes [1]. If we accept this proposition, then it follows that diabetologists and their patients must make "a serious effort to achieve blood glucose levels as close to those in the non-diabetic state as possible"[2], so long as this does not involve an unacceptably high risk of hypoglycaemia or a life style which will damage the patient psychologically.

Until the advent of automatic implantable devices, the only way of controlling insulin dependent diabetes is for the patient to do it himself. Diabetics, unlike patients in most other areas of medicine, have always been given much responsibility for their own treatment and have been expected to carry out tasks such as injections and urine testing which are traditionally the preserve of professionals. In order to control his diabetes successfully the patient needs not only to be educated and motivated, but also to have a reliable means of monitoring his progress so that he can decide when and how to adjust insulin and diet.

As a means of self monitoring, urine tests have always been unsatisfactory. They often do not reflect prevailing blood glucose concentrations accurately [3] but their most serious drawback is that they give no information on blood glucose fluctuations below the level of the renal threshold. This leads patients to equate negative urine tests with impending hypoglycaemia. The problem of the renal threshold also means that urine tests cannot be used to monitor strict control.

Renewed interest in strict diabetic control, the inadequacy of urine testing, and the traditional concept of giving the diabetic patient the responsibility for his own management, have led logically to the development of blood sugar measurement by patients themselves. The idea is not new. As long ago
Table 1. Publications on home blood glucose monitoring

\begin{tabular}{llll}
\hline Authors & System & $\begin{array}{l}\text { Number } \\
\text { of } \\
\text { patients }\end{array}$ & $\begin{array}{l}\text { Age } \\
\text { range } \\
\text { (years) }\end{array}$ \\
\hline Sönksen et al. [8] & Eyetone & 64 & $15-53$ \\
Walford et al. [9] & Reflomat & 69 & $14-65$ \\
Danowski and Sunder [10] & Eyetone & 5 & $11-24$ \\
Peterson et al. [11] & Eyetone & 10 & $\begin{array}{l}\text { Mean 25 } \\
\text { lkeda et al. [12] }\end{array}$ \\
Eyetone & 8 & $18-46$ \\
Skyler et al. [13] & Eyetone & 32 & Not specified \\
\hline
\end{tabular}

as 1962 Keen and Knight [4] showed that their patients could take a sample of capillary blood onto a filter paper which was then delivered to the hospital for analysis. Although this made it possible to measure blood sugars outside hospital, and incidentally showed how poorly controlled most patients were [5], it was unsatisfactory because it did not give an instant results. Similar techniques which do not require the patient to be lent expensive equipment have recently been re-investigated and found to be accurate enough for clinical purposes $[6,7]$. However, any method which does not give an immediate answer is unsatisfactory for the insulin-taking patient who needs to be able to act on the results.

Home blood glucose monitoring has been made possible by the development of glucose oxidase based reagent strips and reflectance meters ${ }^{1}$. Six groups have now reported their experience in teaching insulin treated diabetics to measure their own blood glucose concentrations with a reflectance meter [8--13] (Table 1). Although these trials have differed in detail, the general conclusions have been remarkably similar. In summary these are:

1 Dextrostix-Eyetone system (Ames Corporation), ReflotestReflomat system (Boehringer, Mannheim) 
1. That patients have little difficulty in pricking their fingers.

2. That the results which patients obtain are sufficiently accurate for ordinary clinical practice.

3. That self monitoring of blood glucose levels leads to increased motivation and better control in the majority of patients.

\section{Obtaining the Blood Sample}

Diabetic patients are used to needles and find little difficulty in pricking their fingers. Semi-automatic devices are available [14] but are rarely necessary. Although home blood glucose monitoring has been used for paediatric patients [13] it is probably unreasonable to expect children below the age of 12 to obtain the blood sample themselves. Either blood lancets or disposable needles can be used; patients find either "Monolets" or $3 / 8 \times 26$ gauge disposable needles most satisfactory. Teaching a patient to use a reflectance meter takes about 30 minutes and it is useful for either the doctor or nurse to demonstrate the technique on themselves, since this not only ensures that the machine is working and correctly standardized but also has a considerable psychological impact on the patient.

\section{Accuracy}

Both the Dextrostix-Eyetone and ReflotestReflomat systems give accurate results in the hands of experienced personnel $[15,16]$, although there is some divergence from autoanalyser results when the blood glucose exceeds $12 \mathrm{mmol} / 1(216 \mathrm{mg} / 100 \mathrm{ml})$. Two groups $[9,12]$ have also found a close correlation between blood glucose values obtained by patients themselves and simultaneous laboratory determinations. However, although this shows that patients can use the machine accurately, it does not prove that they will do so in practice. The best guarantee of accuracy lies in careful instruction of patients by someone who is fully conversant with the technique. Although it is probably easier to teach intelligent patients to use a meter, both Sönksen [8] and Walford [9] and their co-workers found that compliance and accuracy were by no means confined to higher socio-economic classes or more intelligent patients.

\section{Improved Motivation and Control}

Direct measurement of blood glucose levels has the great advantage over urine testing that the informa- tion "makes sense" to patients who may previously have performed urine tests conscientiously but without really understanding what they meant. Eighty percent of patients in one series thought that measuring their blood glucose was "a great help" and none found it of no benefit [8]. A high proportion of patients who have experienced home blood glucose monitoring would prefer to continue with it rather than returning to urinanalysis $[8,13]$.

Control has been considerably improved in most patients who have monitored their own blood glucose levels although some of this improvement may be due to the increased interest of both doctors and patients. It is also probable that some of the improvement results from better dietary compliance since many patients who monitor their own blood glucose levels show improved control without changing their insulin dose [13]. In the study of Sönksen et al. [8] the mean clinic blood glucose fell from 11.8 to 8.9 $\mathrm{mmol} / \mathrm{l}$ and two thirds of the patients were able to maintain a level of control in which $80 \%$ of their blood glucose values were below $10 \mathrm{mmol} / 1$ for periods exceeding a year. At the end of the Nottingham study [9] half the patients had glucose profiles in which no more than one of ten blood glucose determinations in a single day exceeded $10 \mathrm{mmol} / \mathrm{l}$. The results achieved by Danowski and Sunder [10] and Peterson et al. [11], albeit on smaller and more selected groups, are even more impressive. In the former study five patients, aged 11 to 25 years, measured their blood glucose levels 23 times a week and were able to maintain almost all values between 3 and $8 \mathrm{mmol} / \mathrm{l}$ for over a year. In the latter study 10 patients made measurements at least six times every day and adjusted their insulin accordingly. The mean $\mathrm{Hb}_{\mathrm{Alc}}$ at the beginning of the study was $10.3 \%$ whereas after four to six months this had fallen to $5.4 \%$.

It has been suggested [17] that strict control is likely to be accompanied by an increased frequency of hypoglycaemic reactions. This has not been the experience in trials of self monitoring of blood glucose. In the St. Thomas's series [8], 34\% of patients had fewer, $46 \%$ the same frequency and $20 \%$ more hypoglycaemic reactions. It seems probable that severe hypoglycaemic reactions, often without warning symptoms, occur most often in patients who have excessive blood glucose fluctuations rather than in patients who are strictly controlled. Self monitoring is a particularly useful means of solving problems in patients who are having hypoglycaemic reactions [9], and is also useful in confirming the presence of a low blood glucose level in patients whose symptoms might be due either to anxiety or hypoglycaemia [13]. Confirmation of hypoglycaemia is easy with a stand- 
ard Dextrostix although with the Boehringer system it is necessary to use a separate stick (Reflotest hypoglycaemie) which has a range from 0.5 to $8 \mathrm{mmol} / \mathrm{l}$.

\section{Frequency of Testing}

How often patients measure their blood sugars depends on the reason for which monitoring is undertaken. In the Nottingham study [9] which was devised primarily to obtain information about and improve the control of brittle diabetics, measurements were made approximately every hour and profiles obtained on two successive days, usually one working and one rest day. Others $[8,13]$ have used measurements four times daily: before each of the main meals and before going to bed. Preprandial or fasting measurements considerably underestimate the size of postprandial blood glucose peaks and are probably insufficient for monitoring the pregnant diabetic or attempting to achieve "super" control in the juvenile diabetic. In these cases the best results seem to be obtained by frequent determinations of blood glucose with multiple injections of insulin. A good compromise for long term monitoring is the protocol advocated by Danowski and Sunder [10] in which patients measured a fasting and one other blood glucose on six out of the seven week days with a full profile on the 7 th day which included determinations in the fasting state, before and after each meal and one blood glucose during the night.

\section{Advantages and Disadvantages of Self Monitoring of Blood Glucose}

The advantages of home blood glucose monitoring are:

1. That it gives information about the pattern of blood glucose fluctuations while patients are going about their ordinary life.

2. It enables the physician to advise on changes of treatment, over the telephone if necessary, on the basis of solid information. It eliminates the need to admit patients to hospital for stabilisation.

3. Patients are motivated and become more active partners in their own management.

Home blood glucose monitoring is complimentary to rather than a substitute for measurement of $\mathrm{Hb}_{\mathrm{Alc}}$. The latter, unlike home blood glucose monitoring, does not depend on the patient's cooperation or accuracy. Furthermore, it provides a way of assessing diabetic control as a single number and is a useful end point at which to direct treatment
[18]. The disadvantage is that it does not indicate how treatment should be changed to obtain better control. Thus, in many ways home blood glucose monitoring and $\mathrm{Hb}_{\mathrm{A} 1 \mathrm{c}}$ measurement each supplement the defects of the other and should be regarded as complimentary investigations. A study using both techniques on the same patients should make it possible to decide how much deviation from normoglycaemia is compatible with a normal $\mathrm{Hb}_{\mathrm{Alc}}$ value.

A potential problem with home blood glucose monitoring is that it might have adverse psychological effects by causing patients to be pre-occupied with measuring their blood glucose levels to the exclusion of a normal life. I have encountered this problem with one patient although it is only fair to say that he had previously been an obsessional urine tester! Generally home blood glucose monitoring seems to improve a patient's, psychological state rather than the converse. Patients who have difficulty in controlling their diabetes often develop a negative self concept and a sense of hopelessness [19]. Home blood glucose monitoring usually leads to increased self confidence and emotional stability which patients attribute to relief at being in control of their own physiology and no longer at the mercy of seemingly random processes [11].

\section{The Future}

The present capital cost of machines and sticks is such that most hospitals will have to use them selectively and sparingly. Home blood glucose monitoring is clearly superior to other methods of assessing dayto-day control and should be regarded as a sine qua non for the management of diabetic pregnancy. For most other diabetic patients a meter is only necessary intermittently to check control or solve particular problems $[9,13]$. If cheaper and more portable machines become available in the future, patients with brittle diabetes, and perhaps even every newly diagnosed insulin taking diabetic, will benefit from doing regular blood test rather than urine tests.

The presently available Reflectance Meters were not designed for patient use and are bulky, mains operated, insufficiently robust and too expensive. The second generation of machines which have recently been marketed are not only cheaper but also more convenient. Both ${ }^{2}$ are portable and have rechargable batteries, a digital display, and built-in timer. If the initial impression that patients prefer

\footnotetext{
2 Glucocheck (Medistron Ltd., Crawley, Sussex, England) and Hypo-Count (Hypoguard, Ipswich, England)
} 
blood to urine tests $[8,13]$ is substantiated, increased production will lead to still smaller and cheaper machines.

\section{Conclusion}

Doctors find it impossible to stabilize and monitor diabetic patients in hospital without measuring blood glucose levels and it seemed logical that patients would also manage themselves better if they were able to measure blood glucose during their ordinary life. The development of glucose oxidase sticks and Reflectance meters has made this possible. Six groups have published their experience with home blood glucose monitoring in 188 patients. All have found that patients have little difficulty in obtaining blood samples and can get accurate results. When patients measure blood rather than urinary glucose they understand the disease better and become more motivated. Control has been greatly improved in the majority of patients and some can maintain normoglycaemia for long periods of time. Cheaper and more portable machines will make the method more widely applicable. Both $\mathrm{Hb}_{\mathrm{A} 1 \mathrm{c}}$ and home blood glucose monitoring will be increasingly used in the management of the insulin-taking diabetic since they provide complementary information.

\section{References}

1. Tchobroutsky, G.: Relation of diabetic control to development of microvascular complications. Diabetologia 15, 143-152 (1978)

2. Cahill, G. F., Etzwiler, D. D., Freinkel, N.: 'Control' and diabetes. N. Engl. J. Med. 294, 1004-1005 (1977)

3. Malone, J. I., Rosenbloom, A. L., Grgic, A., Weber, F. T.: The role of urine sugar in diabetic mangement. Am. J. Dis. Child. 130, 1324-1327 (1976)

4. Keen, H., Knight, R. K.: Self sampling for blood sugar. Lancet 1962 I, 1037-1040

5. Beidas, A.S., Macfie, J. M., Morton, E. B. V., Wells, M. V.: Measurement ot the blood glucose profile in diabetics by a self sampling technique. Paper presented at the 5th Congress of the International Diabetes Federation 1964

6. Wakelin, K., Goldie, D. J., Hartog, M., Robinson, A. P.: Measurement of capillary blood glucose in filter paper spots: An aid to the assessment of diabetic control. Br. Med. J. 1978 II, $468-469$

7. Howe-Davis, S., Holman, R. R., Phillips, M., Turner, R. C.: Home blood sampling for plasma glucose assay in control of diabetes. Br. Med. J. 1978 II, 596-598

8. Sönksen, P. H., Judd, S. L., Lowy, C.: Home monitoring of blood glucose. Lancet 1978 I, 729-732

9. Walford, S., Gale, E. A. M., Allison, S. P., Tattersall, R. B.: Self monitoring of blood glucose. Lancet 1978 I, 732-735

10. Danowski, T. S., Sunder, J. H.: Jet injection of insulin during self monitoring of blood glucose. Diabetes Care 1, 27-33 (1978)

11. Peterson, C. M., Jones, R. L., Dupuis, A., Bernstein, R., O'Shea, M.: Feasibility of tight control of juvenile diabetes through patient monitored glucose determinations. Diabetes 27 [Suppl. 2], 437 (1978)

12. Ikeda, Y., Tajima, N., Nimami, N., Ide, Y., Yoroyama, J., Abe, M.: Pilot study of self measurement of blood glucose using the Dextrostix-Eyetone System for juvenile onset diabetes. Diabetologia 15, 91-93 (1978)

13. Skyler, J.S., Lasky, I. A., Skyler, D. L., Robertson, E. G., Mintz, D. H.: Home blood glucose monitoring as an aid in diabetes management. Diabetes Care 1, 150-157 (1978)

14. Turner, R. C., Holman, R. R.: Automatic lancet for capillary blood sampling. Lancet 1978 II, 712

15. Jarrett, R. J., Keen, H., Hardwick, C.: "Instant" blood sugar measurement using Dextrostix and a reflectance meter. Diabetes 19, 724-726 (1970)

16. Brunton, W. A. T., Steele, J. M., Percy-Robb, I. W.: An assessment of a reflectance meter system for measurement of plasma or blood glucose in the clinic or side ward. Clin. Chim. Acta 75, 359-364 (1977)

17. Siperstein, M. D., Foster, D. W., Knowles, H. C., Levine, R., Maddison, L. L., Roth, J.: Control of blood glucose in diabetic vascular disease. N. Engl. J. Med. 296, 1060-1062 (1977)

18. Gonen, B., Rubenstein, A. H.: Haemoglobin A1 and diabetes mellitus. Diabetologia 15, 1-8 (1978)

19. Bruhn, J. G.: Self concept and the control of diabetes. Am. Fam. Physician 15, 93-97 (1977)

Dr. R. B. Tattersall

Consultant Physician

Nottingham General Hospital

Nottingham NG1 6HA

England 